\title{
Numerical Simulations of Tsunami Generation by Using Boussinesq Equations
}

\author{
X. Zhao, B. L. Wang, H. Liu* \\ Department of Engineering Mechanics, Shanghai Jiao Tong University, Shanghai 200240, China \\ Email: hliu@sjtu.edu.cn
}

\begin{abstract}
Induced by submarine landslides and slumps. Tsunami has become a potential threaten to the safety of the constructions and people along the coastal lines. Correctly predicting the generation and influence of the tsunami becomes more and more important today.

The fully nonlinear and highly dispersive Boussinesq equations [1] are used to study the nonlinear processes of Tsunamis's generation and propagation. To investigate the accuracy of the Boussinesq cquations, linear theory solution of the Laplace equation is taken as target. The method involves Laplace Transform in time and Fouricr Transform in space. Several simple two-dimensional kinematic models of submarine slides and slumps are adopted to simulate the generation of tsunami in horizontal bottom. In order to know whether this method applies to computing smooth bed form of submarine slides, some models of smoothly raised submarine slides are studied. The surface waves computed by the linear wave theory and by the numerical solution of the linearized Boussinesq equations for the case of submarine smoothly raised slides are compared. Good agreements indicate good performance of the proposed model on simulating the beach deformation.

With a specified section across Okinawa trench. scenarios of the Tsunami waveforms induced by bottom deformation, from their generation in deep sea to shallow water, are simulated. Statistical data [2] are used to simulate the sea bottom deformation caused by earthquakes of different magnitude. The relations between the earthquake magnitude and Tsunami waveform are discussed. From their generation site in deep sea to shallow water, the tsunami waveforms have different characteristics. In addition lower magnitude earthquake could results in a wave train of low amplitudes, while high magnitude earthquake results in $\mathrm{N}$-Wave like waves. It is suggested that when the intensity of earthquake increases, the nonlinear and dispersive characteristics are important on simulating the generation and propagating of the earthquake induced Tsunami.

With the Boussinesq model, the wave amplitude is predicted near the coastal line. In the present work, the bottom friction, supposed to be an important dissipation factor for such a long distant propagating, has not yet been considered. To predict nature hazards, the bottom friction needs to be further investigated.
\end{abstract}

\section{REFERENCES}

1. Madsen P. A., Bingham H. B., Liu H. A new Boussinesq method for fully nonlinear waves from shallow to deep water. I Fluid Mech, 2002;462:1-30

2. Ward S. N. Tsunamis. Encyclopedia of Physical Science and Technology. Academic Press, 2002 